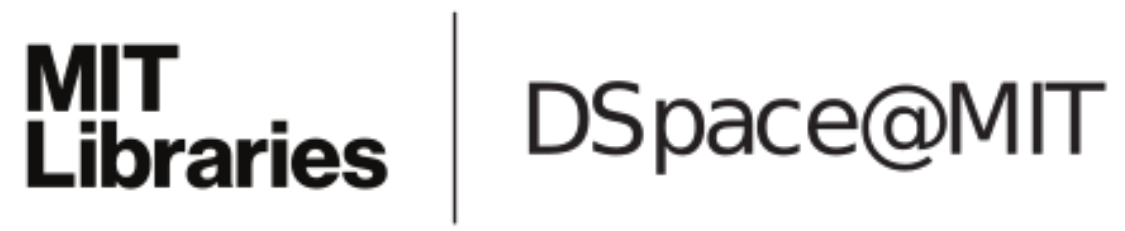

\author{
MIT Open Access Articles
}

\section{Modeling quantum noise for efficient testing of fault-tolerant circuits}

The MIT Faculty has made this article openly available. Please share how this access benefits you. Your story matters.

Citation: Magesan, Easwar et al. “Modeling Quantum Noise for Efficient Testing of Fault-tolerant Circuits." Physical Review A 87.1 (2013). (C2013 American Physical Society

As Published: http://dx.doi.org/10.1103/PhysRevA.87.012324

Publisher: American Physical Society

Persistent URL: http://hdl.handle.net/1721.1/78268

Version: Final published version: final published article, as it appeared in a journal, conference proceedings, or other formally published context

Terms of Use: Article is made available in accordance with the publisher's policy and may be subject to US copyright law. Please refer to the publisher's site for terms of use. 


\title{
Modeling quantum noise for efficient testing of fault-tolerant circuits
}

\author{
Easwar Magesan, ${ }^{1}$ Daniel Puzzuoli, ${ }^{2,3}$ Christopher E. Granade, ${ }^{2,4}$ and David G. Cory ${ }^{2,5,6}$ \\ ${ }^{1}$ Research Laboratory of Electronics, Massachusetts Institute of Technology, Cambridge, Massachusetts 02139, USA \\ ${ }^{2}$ Institute for Quantum Computing, University of Waterloo, Waterloo, Ontario N2L 3G1, Canada \\ ${ }^{3}$ Department of Applied Mathematics, University of Waterloo, Waterloo, Ontario N2L 3G1, Canada \\ ${ }^{4}$ Department of Physics and Astronomy, University of Waterloo, Waterloo, Ontario N2L 3G1, Canada \\ ${ }^{5}$ Department of Chemistry, University of Waterloo, Waterloo, Ontario N2L 3G1, Canada \\ ${ }^{6}$ Perimeter Institute for Theoretical Physics, Waterloo, Ontario N2L 2Y5, Canada
}

(Received 4 July 2012; revised manuscript received 23 October 2012; published 22 January 2013)

\begin{abstract}
Understanding fault-tolerant properties of quantum circuits is important for designing large-scale quantum information processors. In particular, simulating properties of encoded circuits is a crucial tool for investigating the relationship between properties such as the noise model, encoding scheme, and threshold value. For general noisy circuits, these simulations quickly become intractable in the size of the encoded circuit. We introduce a general theoretical method for approximating a noise process by one that allows for efficient Monte Carlo simulation of properties of encoded circuits. The approximation is as close to the original process as possible without overestimating its ability to preserve quantum information, a key property for obtaining honest estimates of threshold values. We numerically illustrate the method with various physically relevant noise models.
\end{abstract}

DOI: 10.1103/PhysRevA.87.012324

PACS number(s): 03.67.Lx, 03.67.Ac, 03.67.Pp

Representing and transforming information using the principles of quantum mechanics implies that a quantum information processor can solve certain problems exponentially faster than currently known classical algorithms [1-3]. Unfortunately, physically realizing such a processor is difficult as quantum systems are extremely sensitive to environmental effects. Hence, one will likely have to take advantage of quantum error-correcting codes [4-7] and fault-tolerance theory [8-10] to perform large-scale quantum computation (QC). In general, one must prove a "threshold theorem" to verify fault-tolerant QC is possible for a given noise model. The main idea is that if the physical gates have an error rate below a threshold $r_{\text {th }}$, and the encoding propagates errors in a controlled manner, then the computational error can be made arbitrarily small by code concatenation.

The ability to Monte Carlo simulate encoded circuits provides valuable information, such as the existence and numerical estimation of $r_{\text {th }}$, and how errors propagate through the encoding [11-16]. In general, these simulations are classically inefficient, and so one often makes assumptions about the types of encoded gates or the noise model, or both. In particular, one often restricts to encoded stabilizer (Clifford) circuits [17]. This is not a significant limitation due to the "magic state" model of QC [18] where Clifford gates, magic states, and standard basis measurements suffice for universal QC. In many important cases [6], the encoded Clifford gates are comprised of physical Clifford gates, and so encoded stabilizer circuits consist only of physical Clifford gates. By the Gottesman-Knill theorem [17], these circuits are efficiently simulatable on a classical computer. Unfortunately, when noise is taken into account, efficient classical simulation is lost. Hence, one often makes assumptions about the noise model at each faulty location, for instance, that the noise is described by a Pauli channel. More generally, when the noise is modeled by a mixed-Clifford channel, classical Monte Carlo simulation is still possible [17]. Pauli channels have various useful properties, which include a simple geo- metric interpretation, closure under composition, and diagonal $\chi$ (process) matrices [19] in the Pauli basis. As well, Pauli channels represent a wide class of physically realistic noise models such as dephasing and depolarizing processes.

In reality, the noise at each location of the circuit will neither be a Pauli nor mixed-Clifford channel. An important question is how one can rigorously approximate the true noise by one of these channels. One method is to remove the off-diagonal elements of the $\chi$ matrix [19], which is attractive since in theory it can be performed via Pauli twirling [20]. Unfortunately, in practice, twirling elements have errors and exact diagonalization can not be achieved. Moreover, as we show later, twirling allows the possibility that many states are better preserved under the twirled channel than the true channel, a nonideal scenario when estimating values of $r_{\text {th }}$.

The goal of this paper is to provide a method to approximate a noise process $\Lambda$ by a channel $\Lambda_{\mathcal{A}}$ such that:

(i) $\Lambda_{\mathcal{A}}$ is as "close" (faithful) to $\Lambda$ as possible,

(ii) $\Lambda_{\mathcal{A}}$ provides an "honest" description of the reliability of $\Lambda$ for preserving quantum information,

(iii) $\Lambda_{\mathcal{A}}$ allows for efficient simulations of properties of quantum circuits.

Point (iii), coupled with the Gottesman-Knill theorem, motivates analyzing the specific cases of $\Lambda_{\mathcal{A}}$ being either a Pauli or mixed-Clifford channel. This will be the focus of this paper, however we note that the theory developed below holds for completely general approximations $\Lambda_{\mathcal{A}}$. Ideally, these $\Lambda_{\mathcal{A}}$ will provide more realistic estimates of the threshold parameter.

To quantify "close" and "honest" we require rigorous methods for comparing quantum states and channels. Two standard methods are derived from the 1 norm on linear operators, \|\|$_{1}$, and the diamond norm on linear superoperators, \|\|$_{\diamond}[21]$. These comparative measures are ideal because they have a clear operational interpretation in terms of maximal distinguishability of quantum states (operations) via POVM measurements. The diamond norm of an arbitrary linear 
superoperator $\mathcal{R}: L\left(\mathbb{C}^{m}\right) \rightarrow L\left(\mathbb{C}^{n}\right)$ is explicitly defined as,

$$
\|\mathcal{R}\|_{\diamond}=\sup _{k \in \mathbb{N}}\left\|\mathcal{R} \otimes \mathcal{I}_{k}\right\|_{1} \text {. }
$$

It is known that the supremum occurs for $k=m$ and so,

$$
\|\mathcal{R}\|_{\diamond}=\left\|\mathcal{R} \otimes \mathcal{I}_{m}\right\|_{1}=\max _{A:\|A\|_{1} \leqslant 1}\left\|\left[\mathcal{R} \otimes \mathcal{I}_{m}\right](A)\right\|_{1}
$$

where $A \in L\left(\mathbb{C}^{m} \otimes \mathbb{C}^{m}\right)$.

Our goal can now be phrased precisely as follows. Suppose $\Lambda$ is a quantum channel whose complete description is given. We want to find the Pauli, or more generally mixed-Clifford, channel $\Lambda_{\mathcal{A}}$ that is the solution to the following constrained optimization problem:

Minimize: $\left\|\Lambda_{\mathcal{A}}-\Lambda\right\|_{\diamond}$

Subject to: for every quantum state $\rho$,

$$
\left\|\left(\Lambda_{\mathcal{A}}-\mathcal{I}\right)(\rho)\right\|_{1} \geqslant\|(\Lambda-\mathcal{I})(\rho)\|_{1} .
$$

When $\Lambda_{\mathcal{A}}$ is a Pauli channel we denote it by $\Lambda_{\mathcal{P}}$ and when it is a mixed-Clifford channel it is denoted by $\Lambda_{\mathcal{C}}$. For any quantum channel $\Lambda$, we call $\|(\Lambda-\mathcal{I})(\rho)\|_{1}$ the "input-output distinguishability" of $\Lambda$ with respect to $\rho$.

We first restrict attention to single-qubit, unital quantum channels and discuss generalizations to multiqubit, nonunital channels later. Thus for now the Hilbert space of the system is given by $\mathcal{H} \equiv \mathbb{C}^{2}$ and $\Lambda(\mathbb{1})=\mathbb{1}$. The single-qubit case is particularly relevant when the noise affecting the circuit is highly local. We note that Ref. [22] has proposed a single-qubit Clifford approximation method that minimizes the HilbertSchmidt (H-S) distance between process matrices, constrained by the average and minimum fidelity to the identity. While the $\mathrm{H}-\mathrm{S}$ norm and average and minimum fidelity constraints are easier to compute, they have no clear operational significance. As well, the state-independent fidelity constraints can lead to underestimation of errors (dishonest approximations), similar to using the Pauli-twirled channel as the approximation (see below).

Our first task is to obtain a general state-independent form for Eq. (3). To begin, we use the Bloch sphere representation [23] where each vector $\vec{r}$ in the unit sphere of $\mathbb{R}^{3}$ is uniquely associated with a quantum state $\rho$, and the boundary of the sphere corresponds to the set of pure states. Quantum channels take a simple form in the Bloch representation [24-26]. Any unital channel $\Lambda$ is uniquely represented by a real matrix $M_{\Lambda}$ such that $\vec{r} \rightarrow M_{\Lambda} \vec{r}$. This representation has many intuitive features, for instance a unitary operation $\mathcal{U}$ is represented by an orthogonal matrix $M_{\mathcal{U}}$ and Pauli channels are diagonal.

We exploit a useful correspondence relating the 1-norm distance between states $\rho_{1}, \rho_{2}$ and the standard 2-norm (Euclidean) distance between their Bloch vectors $\vec{r}_{1}, \vec{r}_{2}$,

$$
\left\|\rho_{1}-\rho_{2}\right\|_{1}=\left\|\vec{r}_{1}-\vec{r}_{2}\right\|_{2} \text {. }
$$

Equation (4) allows for a state-independent version of Eq. (3). Indeed, Eq. (4) implies Eq. (3) is equivalent to

$$
\left\|\vec{r}-M_{\mathcal{A}} \vec{r}\right\|_{2} \geqslant\left\|\vec{r}-M_{\Lambda} \vec{r}\right\|_{2}
$$

holding for all unit vectors $\vec{r}$. Using the theory of quadratic forms, Eq. (5) is equivalent to $A \geqslant B$ where

$$
\begin{aligned}
A & :=\left(\mathbb{1}-M_{\mathcal{A}}\right)^{T}\left(\mathbb{1}-M_{\mathcal{A}}\right), \\
B & :=\left(\mathbb{1}-M_{\Lambda}\right)^{T}\left(\mathbb{1}-M_{\Lambda}\right),
\end{aligned}
$$

and $T$ is the transpose. In the case of a Pauli approximation, $A=\left(\mathbb{1}-M_{\mathcal{P}}\right)^{2}$. Since a description of $\Lambda$ is assumed to be given, $B$ is straightforward to compute [27]. Thus, finding entries of $M_{\mathcal{A}}$ which give $A-B \geqslant 0$ will ensure Eq. (3) is satisfied. Minimizing $\left\|\Lambda_{\mathcal{A}}-\Lambda\right\|_{\diamond}$ over possible values gives the solution to our problem.

We first look at Pauli approximations and then, using intuition from these examples, discuss mixed-Clifford approximations. Before analyzing the results in detail, let us set notation. For a single qubit, $\mathcal{P}_{1}:=\left\{\sigma_{0}, \sigma_{1}, \sigma_{2}, \sigma_{3}\right\}=\{\mathbb{1}, X, Y, Z\}$ is the usual orthogonal Pauli basis for the set of $2 \times 2$ complex matrices. The multiqubit Pauli basis $\mathcal{P}_{n}$ is obtained by taking tensor products of elements of $\mathcal{P}_{1} . \mathcal{P}_{n}$ consists of traceless (except for $\mathbb{1}$ ), unitary and Hermitian matrices, and is a group when one includes phases. The $n$-qubit Clifford group Clif $_{n}$ is defined to be the normalizer of $\mathcal{P}_{n}$ and can be generated by the Hadamard $(H)$, phase $(S)$ and CNOT gates applied on pairs of qubits (note $\mathcal{P}_{n} \subset \mathrm{Clif}_{n}$ ). A single-qubit mixed-Clifford channel $\Lambda_{\mathcal{C}}$ has the form $\Lambda_{\mathcal{C}}(\rho)=\sum_{i} p_{i} C_{i} \rho C_{i}^{\dagger}$ where $\left\{p_{i}\right\}$ is a probability distribution, the $\left\{C_{i}\right\}$ form a subset of Clif ${ }_{1}$, and $\rho$ is an arbitrary mixed state. For a Pauli channel, $\Lambda_{\mathcal{P}}(\rho)=\sum_{i=0}^{3} p_{i} \sigma_{i} \rho \sigma_{i}$.

Numerical results. We apply the approximation scheme to four types of channels. The first three are well-known examples of unital channels and have a closed-form representation. These simple examples provide a clear understanding of the details of our method. The fourth channel does not have a closed-form description and the goal of this example is to demonstrate the applicability of the method even when no structural information is available.

The three simple unital channels are given below:

$$
\begin{gathered}
\Lambda^{(1)}(\rho)=(1-p) \rho+p\left(\vec{n}_{p} \cdot \vec{\sigma}\right) \rho\left(\vec{n}_{p} \cdot \vec{\sigma}\right), \\
\Lambda^{(2)}(\rho)=(1-3 p) \rho+p \sum_{i=1}^{3} \sigma_{i} \rho \sigma_{i}, \\
\Lambda^{(3, k)}(\rho)=\exp \left(-i \frac{\theta}{2} \vec{n}_{k} \cdot \vec{\sigma}\right) \rho \exp \left(i \frac{\theta}{2} \vec{n}_{k} \cdot \vec{\sigma}\right) .
\end{gathered}
$$

The parameter values we choose are $p=0.01$, $\vec{n}_{p}=[\sin (\pi / 8), 0, \cos (\pi / 8)], \quad \theta=0.02, \quad$ and $\vec{n}_{k}=$ $\left[\sin \left(\theta_{k}\right), 0, \cos \left(\theta_{k}\right)\right]$ where $\theta_{k}=\frac{k \pi}{8}$ for $k \in\{0,1,2\} . \Lambda^{(1)}(\rho)$ represents dephasing noise about a non-Pauli axis, $\Lambda^{(2)}(\rho)$ represents a depolarizing channel and the $\Lambda^{(3, k)}(\rho)$ represent rotations about axes in the $x-z$ plane starting from the $z$ axis and ending at the $x$ axis. The depolarizing channel was included to verify that when $\Lambda$ is a Pauli channel, the scheme returns $\Lambda_{\mathcal{P}}=\Lambda$.

The $\chi$-matrix for the structureless fourth channel, $\Lambda^{(a)}$, was obtained via numerical simulation of an arbitrary noise process,

$$
\chi^{(a)} \approx\left[\begin{array}{cccc}
0.9942 & 0.0051 i & -0.0058 i & 0.0048 i \\
-0.0051 i & 0.0013 & 0.0020 & 0.0013 \\
0.0058 i & 0.0020 & 0.0032 & 0.0020 \\
-0.0048 i & 0.0013 & 0.0020 & 0.0013
\end{array}\right]
$$

Clearly this channel is not Pauli and it does not have any obvious structure that can be taken advantage of. 
TABLE I. Pauli channel approximations $\Lambda_{\mathcal{P}}^{(i)}$ and diamond-norm distance between $\Lambda_{\mathcal{P}}^{(i)}$ and $\Lambda^{(i)}$.

\begin{tabular}{lccccc}
\hline \hline & \multicolumn{5}{c}{ Approximation $\Lambda_{\mathcal{P}}^{(i)}$} \\
\cline { 2 - 5 } Channel & {$\left[\chi_{\mathcal{P}}\right]_{0,0}$} & {$\left[\chi_{\mathcal{P}}\right]_{1,1}$} & {$\left[\chi_{\mathcal{P}}\right]_{2,2}$} & {$\left[\chi_{\mathcal{P}}\right]_{3,3}$} & $\left\|\Lambda_{\mathcal{P}}^{(i)}-\Lambda^{(i)}\right\|_{\odot}$ \\
\hline$\Lambda^{(1)}$ & 0.9860 & 0.0020 & 0.0040 & 0.0080 & 0.0152 \\
$\Lambda^{(2)}$ & 0.9700 & 0.0100 & 0.0100 & 0.0100 & 0 \\
$\Lambda^{(3,0)}$ & 0.9900 & 0 & 0 & 0.0100 & 0.0281 \\
$\Lambda^{(3,1)}$ & 0.9860 & 0.0022 & 0.0040 & 0.0078 & 0.0359 \\
$\Lambda^{(3,2)}$ & 0.9850 & 0.0050 & 0.0050 & 0.0050 & 0.0381 \\
$\Lambda^{(a)}$ & 0.9827 & 0.0032 & 0.0067 & 0.0074 & 0.0310 \\
\hline \hline
\end{tabular}

$\Lambda_{\mathcal{P}}$ for each case is given in Table I via its $\chi$-matrix $\chi_{\mathcal{P}}$. The diamond-norm distance for each case is also given and was calculated using the semidefinite program of Ref. [28].

We give $\chi$-matrix values rather than Bloch-matrix values because each can be obtained from the other straightforwardly [27], the $\chi$ matrix is a standard tool in process tomography [19], and $\chi$-matrix entries can be efficiently estimated via experiments [29-35]. In particular, for any $\Lambda, \chi_{0,0}$ is directly related to the average fidelity of $\Lambda$ [36], which is a standard figure of merit for the quality of an intended unitary (identity) operation. The $\chi_{0,0}$ elements of $\Lambda^{(1)}, \Lambda^{(2)}$, and the $\Lambda^{(3, j)}$ are given by $0.9900,0.9700$, and 0.9999 respectively.

As expected, from the constraint of Eq. (3), $\left[\chi_{\mathcal{P}}\right]_{0,0}^{(i)} \leqslant \chi_{0,0}^{(i)}$ in all cases. As well, since $\Lambda^{(2)}$ is depolarizing, $\Lambda_{\mathcal{P}}^{(2)}=\Lambda^{(2)}$, which verifies our consistency check. For the unitary rotations $\Lambda^{(3, k)}$, the decrease in average fidelity is relatively large and is a maximum at $k=2$. When $k=0, \Lambda_{\mathcal{P}}^{(3,0)}$ is dephasing about the $z$ axis, and as the rotation axis angle approaches $\frac{\pi}{4}$, the approximation becomes depolarizing. Note that by symmetry, as the rotation axis goes to $\frac{\pi}{2}$, the approximation will again be dephasing (with the roles of $x$ and $z$ reversed).

It is straightforward to show there is a dephasing channel that exactly reproduces the input-output distinguishability of a unitary rotation about any axis. Indeed, for $\Lambda^{(3,0)}$ with rotation angle $\theta$ and input state is $\rho$ (which has Bloch vector $\vec{r}$ at an angle $\alpha$ relative to $z$ ),

$$
\left\|\left(\Lambda^{(3,0)}-\mathcal{I}\right)(\rho)\right\|_{1}=2\left|\sin \left(\frac{\theta}{2}\right)\right||\sin (\alpha)|\|\vec{r}\|_{2} .
$$

As well, for the dephasing channel $\Lambda_{\mathcal{D}}^{(3,0)}$ about $z$ given by $\Lambda_{\mathcal{D}}^{(3,0)}(\rho)=(1-p) \rho+p \sigma_{z} \rho \sigma_{z}$, the input-output distinguishability has a very similar form,

$$
\left\|\left(\Lambda_{\mathcal{D}}^{(3,0)}-\mathcal{I}\right)(\rho)\right\|_{1}=2 p|\sin (\alpha)|\|\vec{r}\|_{2} .
$$

Hence, setting $p=|\sin (\theta / 2)|$ implies $\Lambda_{\mathcal{D}}^{(3,0)}$ exactly matches the input-output distinguishability of $\Lambda^{(3,0)}$ (this dephasing channel is the optimal channel found by our algorithm). By unitary invariance, a rotation about any axis $\hat{n}$ and a dephasing channel about $\hat{n}$ have the same input-output distinguishability if $p=|\sin (\theta / 2)|$.

For each $j$, we use the Bloch sphere to visualize the difference between $\Lambda_{\mathcal{P}}^{(3, j)}$ and the above dephasing channel that exactly matches its input-output distinguishability for each $\rho$. We denote these dephasing channels by $\Lambda_{\mathcal{D}}^{(3, j)}$. Figure 1 contains plots of the deformation of the Bloch sphere in the
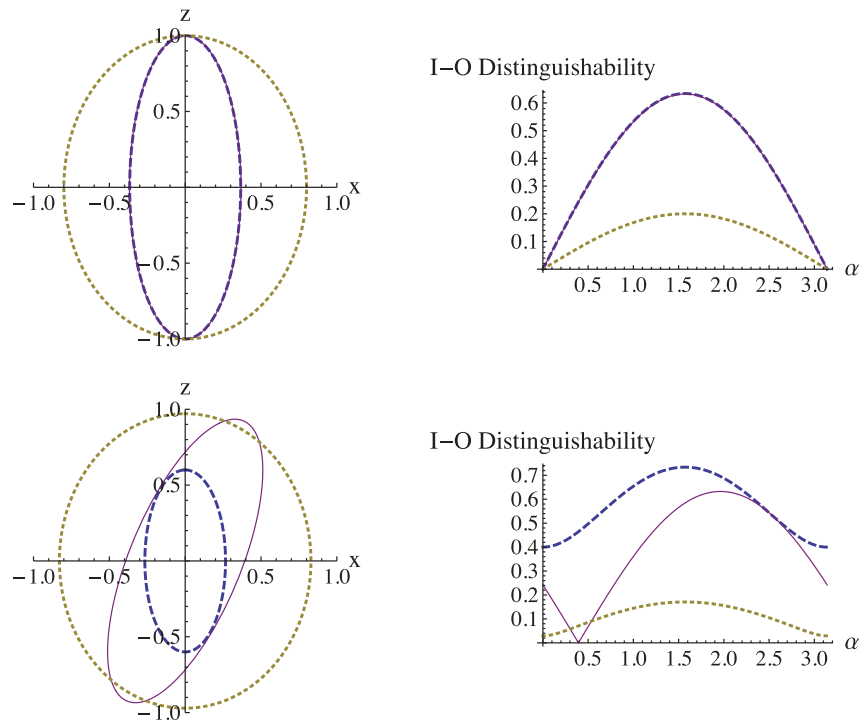

I-O Distinguishability
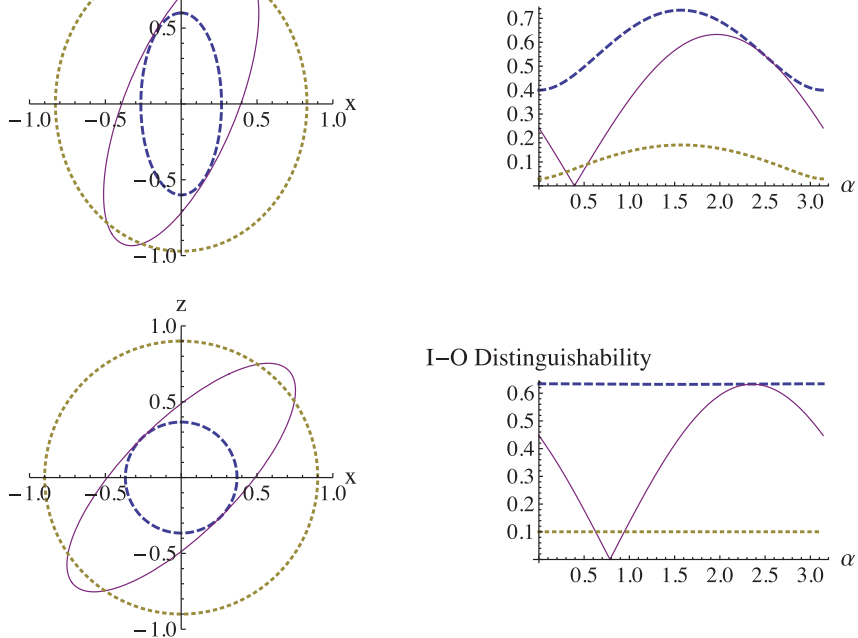

I-O Distinguishability

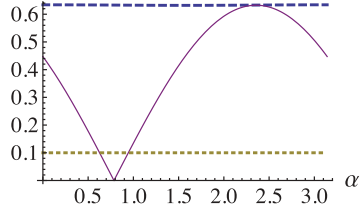

FIG. 1. (Color online) $\Lambda_{\mathcal{P}}^{(3, j)}$ (dashed blue line), $\Lambda_{\mathcal{D}}^{(3, j)}$ (solid red line), $\Lambda_{t}^{(3, j)}$ (dotted gold line) for $j=0,1,2$. Left column: Action on the $x-z$ plane of the Bloch sphere. Right column: Input-output distinguishability for states $\rho$ at angle $\alpha$ relative to $z$ axis.

$x-z$ plane by $\Lambda_{\mathcal{P}}^{(3, j)}$ (dashed blue line) and $\Lambda_{\mathcal{D}}^{(3, j)}$ (solid red line) for $j=0,1,2$. To enhance the visualization we rotate by a larger angle, $\theta=2 \sin ^{-1}(\sqrt{0.1})$.

Clearly $\Lambda_{\mathcal{P}}^{(3, j)}$ diverges from $\Lambda_{\mathcal{D}}^{(3, j)}$ as $\theta_{j}$ goes to $\frac{\pi}{4}$. Hence, the best Pauli approximation becomes significantly worse than the best dephasing approximation. This indicates it can be useful to search over more general classes of channels than Pauli channels. For instance if we augment $\mathcal{P}_{1}$ with the Hadamard gate $H$ then at $j=2$ our approximation $\Lambda_{\mathcal{A}}$ is a mixed-Clifford channel and is exactly $\Lambda_{\mathcal{D}}^{(3,2)}$. This is because $\Lambda_{\mathcal{D}}^{(3,2)}$ has the form

$$
\Lambda_{\mathcal{D}}^{(3,2)}(\rho)=(1-p) \rho+p H \rho H,
$$

where $p=|\sin (\theta / 2)|=\sqrt{0.1}$. Thus knowing the form of the noise $\Lambda$ can provide intuition as to what gates can be augmented to $\mathcal{P}_{1}$ to obtain a better approximation.

Let us now compare some of the Pauli approximations of Table I with those that would be obtained from twirling over the Pauli group. For any $\Lambda$ we denote its Pauli twirl by $\Lambda_{t}$. We analyze how $\Lambda_{t}^{(i)}$ and $\Lambda_{\mathcal{P}}^{(i)}$ compare in terms of our two conditions for an optimal approximation: first, how $\left\|\Lambda_{\mathcal{P}}^{(i)}-\Lambda^{(i)}\right\|_{\diamond}$ compares to $\left\|\Lambda_{t}^{(i)}-\Lambda^{(i)}\right\|_{\diamond}$ for $i=1,(3,0),(3,1),(3,2), a$ (see Table II), and second, how $\left\|\left(\Lambda_{\mathcal{P}}^{(3, j)}-\mathcal{I}\right)(\rho)\right\|_{1}$ and $\|\left(\Lambda_{t}^{(3, j)}-\right.$ $\mathcal{I})(\rho) \|_{1}$ compare for $j=0,1,2$ and states $\rho$ at angle $\alpha$ relative to $z$ (see right-hand side of Fig. 1). 
TABLE II. Comparisons of $\left\|\Lambda^{(i)}-\Lambda_{t}^{(i)}\right\|_{\diamond}$ vs $\left\|\Lambda^{(i)}-\Lambda_{\mathcal{P}}^{(i)}\right\|_{\diamond}$.

\begin{tabular}{lcc}
\hline \hline Channel & $\left\|\Lambda^{(i)}-\Lambda_{t}^{(i)}\right\|_{\diamond}$ & $\left\|\Lambda^{(i)}-\Lambda_{\mathcal{P}}^{(i)}\right\|_{\diamond}$ \\
\hline$\Lambda^{(1)}$ & 0.0071 & 0.0152 \\
$\Lambda^{(3,0)}$ & 0.0020 & 0.0281 \\
$\Lambda^{(3,1)}$ & 0.0020 & 0.0359 \\
$\Lambda^{(3,2)}$ & 0.0020 & 0.0381 \\
$\Lambda^{(a)}$ & 0.0230 & 0.0310 \\
\hline \hline
\end{tabular}

We see that for each $i,\left\|\Lambda^{(i)}-\Lambda_{t}^{(i)}\right\|_{\diamond} \leqslant\left\|\Lambda^{(i)}-\Lambda_{\mathcal{P}}^{(i)}\right\|_{\diamond}$ and so the Pauli twirl better models the original channel. However from Fig. 1 we see that for each $j$, except for a small set of states, $\left\|\left(\Lambda_{\mathcal{P}}^{(3, j)}-\mathcal{I}\right)(\rho)\right\|_{1} \geqslant\left\|\left(\Lambda^{(3, j)}-\mathcal{I}\right)(\rho)\right\|_{1} \geqslant$ $\left\|\left(\Lambda_{t}^{(3, j)}-\mathcal{I}\right)(\rho)\right\|_{1}$. Hence, the truncation underestimates the error whereas our channel does not, as designed. So, for the price of a slightly larger diamond-norm distance to the true error $\Lambda$, we have ensured that we do not underestimate the error. This is crucial when obtaining estimates of the threshold parameter.

For $\Lambda^{(a)}$, we also compute the diamond norms $\| \Lambda_{t}^{(a)}-$ $\mathcal{I}\left\|_{\diamond}=0.0116,\right\| \Lambda^{(a)}-\mathcal{I} \|_{\diamond}=0.0248$, and $\left\|\Lambda_{\mathcal{P}}^{(a)}-\mathcal{I}\right\|_{\diamond}=$ 0.0347 , which measure the distance of each channel to the ideal "no-operation" channel. These give the expected relationships,

$$
\begin{aligned}
\left\|\Lambda_{t}^{(a)}-\Lambda^{(a)}\right\|_{\diamond} & <\left\|\Lambda_{\mathcal{P}}^{(a)}-\Lambda^{(a)}\right\|_{\diamond}, \\
\left\|\Lambda_{t}^{(a)}-\mathcal{I}\right\|_{\diamond} & <\left\|\Lambda^{(a)}-\mathcal{I}\right\|_{\diamond}<\left\|\Lambda_{\mathcal{P}}^{(a)}-\mathcal{I}\right\|_{\diamond} .
\end{aligned}
$$

We now discuss generalizing to the nonunital and multiqubit cases. The Bloch sphere representation of a nonunital single-qubit channel $\Lambda$ is given by a matrix $M_{\Lambda}$ and vector $\vec{t}$ that represents the nonunitality

$$
\vec{r} \mapsto M_{\Lambda} \vec{r}+\vec{t}
$$

One can show using a similar quadratic form argument that if $A \geqslant B$ where

$$
\begin{gathered}
A:=\left(\mathbb{1}-M_{\mathcal{A}}\right)^{T}\left(\mathbb{1}-M_{\mathcal{A}}\right), \\
B:=\left(\mathbb{1}-M_{\Lambda}\right)^{T}\left(\mathbb{1}-M_{\Lambda}\right)+\left(\|\vec{t}\|_{2}^{2}+2\|\vec{v}\|_{2}\right) \mathbb{1},
\end{gathered}
$$

and $\vec{v}$ is given by $\vec{v}=\left(\mathbb{1}-M_{\Lambda}\right)^{T} \vec{t}$, then for every unit vector $\vec{r}$

$$
\left\|\vec{r}-M_{\mathcal{A}} \vec{r}\right\|_{2} \geqslant\left\|\vec{r}-\left(M_{\Lambda} \vec{r}+\vec{t}\right)\right\|_{2} .
$$

Hence, for every pure state $\rho$, Eq. (3) is satisfied. Thus, similarly to the unital case, minimizing $\left\|\Lambda_{\mathcal{A}}-\Lambda\right\|_{\diamond}$ over all possible $\Lambda_{\mathcal{A}}$ satisfying $A \geqslant B$ gives the pure-state solution to our problem.
TABLE III. Pauli channel approximation details for $\Lambda^{(2 q)}$.

\begin{tabular}{lccc}
\hline \hline \multirow{2}{*}{ Channel } & \multicolumn{2}{l}{ Approximation $\Lambda_{\mathcal{P}}^{(2 q)}$} & \\
\cline { 2 - 3 } & $\chi_{00}$ & $\chi_{x x}$ & $\left\|\Lambda_{\mathcal{P}}^{(2 q)}-\Lambda^{(2 q)}\right\|_{\diamond}$ \\
\hline$\Lambda^{(2 q)}$ & 0.9900 & 0.0100 & 0.0281 \\
\hline \hline
\end{tabular}

For the multiqubit case, it is not true in general that for states $\rho_{1}$ and $\rho_{2},\left\|\rho_{1}-\rho_{2}\right\|_{1}=\left\|\vec{r}_{1}-\vec{r}_{2}\right\|_{2}$, however it is still likely the case that if $A \geqslant B$ then Eq. (3) is satisfied. The multiqubit case is of significance when considering correlated noise models in encoded circuits. Here we numerically analyze the collective unitary noise model $\Lambda^{(2 q)}$ with Kraus operator $\exp \left(-i 0.01 \sigma_{X} \otimes \sigma_{X}\right)$ The $\chi$ matrix is $16 \times 16$ but, since it only contains $\mathbb{1} \otimes \mathbb{1}$ and $\sigma_{x} \otimes \sigma_{x}$ terms, it is sparse and can be compressed to

$$
\chi^{(2 q)}=\left[\begin{array}{cc}
0.9999 & 0.0100 i \\
-0.0100 i & 0.0001
\end{array}\right] .
$$

The Pauli approximation for this channel, $\Lambda_{\mathcal{P}}^{(2 q)}$, is given in Table III. As expected, $\Lambda_{\mathcal{P}}^{(2 q)}$ is symmetric across both qubits and gives the exact same results as for the single-qubit rotation about $\sigma_{X}, \Lambda_{\mathcal{P}}^{(3,4)}$, given in Table I.

To conclude, we have provided a method for obtaining optimal honest mixed-Clifford approximations of quantum channels. We explicitly analyzed single-qubit Pauli approximations, discussed the extension to mixed-Clifford approximations, and showed that alternative twirling methods lead to highly dishonest approximations. We have presented a rigorous extension to the nonunital case and have provided numerical evidence that our method likely holds in the multiqubit case. These results are essential for simulating quantum circuits as they allow for more honest estimates of threshold values as well as scalable simulation of circuit properties.

We thank Joseph Emerson, Daniel Gottesman, Ian Hincks, and Marcus Silva for helpful discussions, and acknowledge support from NSERC, CIFAR, CERC, and the Ontario government. This work was partially supported by the Intelligence Advanced Research Projects Activity (IARPA) via Department of Interior National Business Center, Contract No. DllPC20166. The US Government is authorized to reproduce and distribute reprints for Governmental purposes notwithstanding any copyright annotation thereon. Disclaimer: The views and conclusions contained herein are those of the authors and should not be interpreted as necessarily representing the official policies or endorsements, either expressed or implied, of IARPA, DoI/NBC, or the US Government.
[1] P. W. Shor, in Proceedings of the 35th Annual Symposium on Foundations of Computer Science (FOCS) (IEEE Press, Los Alamitos, CA, 1994), pp. 124-134.

[2] R. Feynman, Int. J. Theor. Phys. 21, 6 (1982).

[3] L. K. Grover, 28th Annual ACM Symposium on the Theory of Computing, 1996 (unpublished).
[4] P. W. Shor, Phys. Rev. A 52, R2493 (1995).

[5] A. R. Calderbank and P. W. Shor, Phys. Rev. A 54, 1098 (1996).

[6] A. Steane, Proc. Roy. Soc. London A 452, 2551 (1996).

[7] E. Knill, R. Laflamme, and L. Viola, Phys. Rev. Lett. 84, 2525 (2000). 
[8] D. Aharonov and M. Ben-Or, in Proceedings of the 29th Annual ACM Symposium on Theory of Computing, 1997 (unpublished).

[9] E. Knill, R. Laflamme, and W. Zurek, Proc. R. Soc. Lond. A 454, 365 (1997).

[10] J. Preskill, arXiv:quant-ph/9712048.

[11] C. Zalka, arXiv:quant-ph/9612028v2.

[12] A. M. Steane, Phys. Rev. A 68, 042322 (2003).

[13] P. Aliferis, D. Gottesman, and J. Preskill, Quantum Inf. Comp. 6, 97 (2006).

[14] D. C. Marinescu and G. M. Marinescu, in Proceedings of the 22nd Workshop on Principles of Advanced and Distributed Simulation, PADS '08 (IEEE Computer Society, Washington, DC, USA, 2008), p. 152.

[15] A. W. Cross, D. P. DiVincenzo, and B. M. Terhal, Quantum Inf. Comp. 9, 0541 (2009).

[16] M. Whitney, Ph.D. thesis, University of California, Berkeley, 2009.

[17] D. Gottesman, Ph.D. thesis, California Institute of Technology, 1997.

[18] S. Bravyi and A. Kitaev, Phys. Rev. A 71, 022316 (2005).

[19] I. L. Chuang and M. A. Nielsen, J. Mod. Opt. 44, 2455 (1997).

[20] M. Silva, E. Magesan, D. W. Kribs, and J. Emerson, Phys. Rev. A 78, 012347 (2008).
[21] A. Kitaev, Russian Mathematical Surveys 52, 1191 (1997).

[22] M. Gutierrez et al., arXiv:1207.0046.

[23] F. Bloch, Phys. Rev. 70, 460 (1946).

[24] P. S. Bourdon and H. T. Williams, Phys. Rev. A 69, 022314 (2004).

[25] A. Fujiwara and P. Algoet, Phys. Rev. A 59, 3290 (1999).

[26] M. B. Ruskai, S. Szarek, and E. Werner, Linear Algebr. Appl. 347, 159 (2002).

[27] M. A. Nielsen and I. L. Chuang, Quantum Computation and Information (Cambridge University Press, Cambridge, 2000).

[28] J. Watrous, Theory of Computing 5, 217 (2009).

[29] M. Mohseni and D. A. Lidar, Phys. Rev. Lett. 97, 170501 (2006).

[30] E. Knill et al., Phys. Rev. A 77, 012307 (2008).

[31] J. Emerson et al., Science 317, 1893 (2007).

[32] E. Magesan, J. M. Gambetta, and J. Emerson, Phys. Rev. Lett. 106, 180504 (2011).

[33] E. Magesan et al., Phys. Rev. Lett. 109, 080505 (2012).

[34] A. Bendersky, F. Pastawski, and J. P. Paz, Phys. Rev. Lett. 100, 190403 (2008).

[35] A. Shabani et al., Phys. Rev. Lett. 106, 100401 (2011).

[36] M. Nielsen, Phys. Lett. A 303, 249 (2002). 